Research Article

\title{
Investigation of the Magnetic Properties of Ferrites in the CoO-NiO-ZnO Using Simplex-Lattice Design
}

\author{
Liliya Frolova $\mathbb{D}^{1}$ and Oleg Khmelenko ${ }^{2}$ \\ ${ }^{1}$ Department of Inorganic Materials Technology and Ecology, Ukrainian State University of Chemical Technology, \\ Dnepr/49005, Ukraine \\ ${ }^{2}$ Department Radiophysics, Oles Honchar Dnipro National University, Dnepr/49000, Ukraine
}

Correspondence should be addressed to Liliya Frolova; 19kozak83@gmail.com

Received 29 August 2018; Accepted 26 October 2018; Published 19 December 2018

Guest Editor: Anil Annadi

Copyright (c) 2018 Liliya Frolova and Oleg Khmelenko. This is an open access article distributed under the Creative Commons Attribution License, which permits unrestricted use, distribution, and reproduction in any medium, provided the original work is properly cited.

\begin{abstract}
The article is devoted to the analysis of changes in the magnetic characteristics of ferrites in the $\mathrm{CoO}-\mathrm{NiO}-\mathrm{ZnO}$ system by the simplex method. Ferrites of Ni-Zn, Co-Zn, and Co-Ni were synthesized in the form of nanoparticles (20-40 nm) using a new method for processing contact nonequilibrium low-temperature plasma (CNP). The effect of the mutual influence of the contents of different cations on the saturation magnetization and the coercive field was investigated using the simplexlattice method. A magnetic investigation using a vibrational magnetometer shows that low magnetization values are observed for $\mathrm{Ni}-\mathrm{Zn}$ ferrites and high for the entire $\mathrm{Co}-\mathrm{Zn}$ and $\mathrm{Co}-\mathrm{Ni}$ ferrite series. EPR spectra show that the value of the resonant field and line width corresponds to the value of magnetic saturation and is due to the arrangement of cations on sublattices.
\end{abstract}

\section{Introduction}

Oxides of composition $\mathrm{MeFe}_{2} \mathrm{O}_{4}\left(\mathrm{Me}-\mathrm{Ni}^{2+}, \mathrm{Co}^{2+}, \mathrm{Zn}^{2+}\right)$ have important technological properties. For example, ferrites of transition metals with a spinel structure are used as magnetic electrical materials $[1,2]$ catalysts of a number of reactions $[3,4]$. Technological operations for the synthesis of such compounds require, as a rule, the use of high-temperature heat treatment and complex hardware. In this case, both traditional ceramic (from metal oxides) methods and novel technologies are used. For example, in the synthesis of nickel (II) ferrite, cobalt (II) and zinc (II), and hydrothermal methods [5], microwave treatment [6] is used. The attention of chemists is concentrated on the development of new methods for obtaining ferrites of transition materials with a given set of properties. A characteristic trend of recent times is the development of technologies for obtaining nanodispersed ferrites.
It is known that nanosized spinel ferrites exhibit properties and phenomena that cannot be explained on the basis of the structure and properties of the consolidated substance $[1,2]$. Thus, the transition of ferrites of transition metals to a nanoscale state is accompanied by a significant change in their magnetic properties (coercive field, magnetization magnitudes, crystallographic anisotropy, and Curie temperature). And their properties essentially depend on the technology of obtaining samples. The authors of [7] point out that the effect of size effects in the synthesis of nanoscale ferrospinels by coprecipitation of salt solutions with the use of additional high-energy short-term exposures is much stronger than in the case of using traditional technologies. The creation of adequate models of the magnetic state of such materials is one of the urgent problems of materials science. This is due both to the wide possibilities of their practical use and to the need to develop theoretical ideas about the effect of dimensional and surface effects on magnetic 
properties $[8,9]$. There is no unified theory that explains the variation of magnetic properties over a wide range. At present, there are basic theories. The "shell" model gives a qualitative explanation of the effect of decreasing magnetization with decreasing particle sizes. Neel's theory establishes the dependence of the magnetization on the distribution of cations over the sublattices. A theory is known [10] about the formation of the magnetic properties of nanosized ferrimagnets due to the anisotropy induced by internal elastic microstrains.

The aim of this work is to establish the relationship between the magnetic characteristics of ferrites of the composition $\mathrm{MeFe}_{2} \mathrm{O}_{4}(\mathrm{Me}-\mathrm{Ni}, \mathrm{Co}, \mathrm{Zn})$ and structural characteristics obtained by processing a contact nonequilibrium low-temperature plasma.

\section{Materials and Methods}

In order to reduce energy consumption, temperature, and time of synthesis in the production of ferrites of different composition, in this work, a method of precipitation of hydroxides was used, followed by treatment of the suspension with CNP, washing, and drying.

Reagent grade $\mathrm{FeSO}_{4} 7 \mathrm{H}_{2} \mathrm{O}, \mathrm{NiSO}_{4} 7 \mathrm{H}_{2} \mathrm{O}, \mathrm{CoSO}_{4} 7 \mathrm{H}_{2} \mathrm{O}$, and $\mathrm{ZnSO}_{4} 7 \mathrm{H}_{2} \mathrm{O}$ were used as the starting materials.

The hydroxide sol which was obtained by alkali precipitation was treated with contact low-temperature nonequilibrium plasma in a laboratory plasma chemical plant, which consists of a single-stage plasma reactor of a discrete type, a step-up transformer, an ignition transformer, and a vacuum pump. After treatment, the resulting precipitate was washed and dried for further investigation. Plasmachemical treatment of suspensions was carried out in a gas-liquid plasma-chemical reactor of periodic action. The reactor is made of glass and is equipped with an external jacket for thermostating the medium to be treated. Electrodes of stainless steel are placed in the lower and upper part of the reactor. $40 \mathrm{~cm}^{3}$ of slurry was poured into the reactor; the anode position was adjusted so that the distance between its lower base and the surface of the liquid was $10.0 \mathrm{~mm}$. The plasma column formed as a result of breakdown was a tool for processing. To obtain a plasma discharge, the pressure in the reactor was maintained at $0.08 \mathrm{MPa}$. Electrodes were supplied with a direct current with a voltage in the range of $500-600 \mathrm{~V}$, the value of which was varied so that the current strength in the circuit was 100-150 mA.

$\mathrm{X}$-ray diffraction patterns of the pigments were obtained on a DRON-2.0 instrument in monochromatized $\mathrm{Co}_{\alpha}$ radiation. The lattice parameter was calculated from the SelyakovScherrer equation.

The determination of the magnetic characteristics was carried out using a vibration magnetometer. A change in the solution medium was observed at regular intervals using a $\mathrm{pH}$ meter-pH-150 MI. EPR spectra were obtained using a Radiopan SE/X-2543 radio spectrometer. The signal strength, the resonant magnetic field, and the signal width were used to characterize the ESR signals.
TABle 1: Matrix planning of the simplex-lattice design $\{3,3\}$.

\begin{tabular}{lcccc}
\hline № & $\mathrm{Co}$ & $\mathrm{Ni}$ & $\mathrm{Zn}$ & $y_{i}$ \\
\hline 1 & 1.0 & 0.0 & 0.0 & $y_{1}$ \\
2 & 0.0 & 1.0 & 0.0 & $y_{2}$ \\
3 & 0.0 & 0.0 & 1.0 & $y_{3}$ \\
4 & 0.333 & 0.667 & 0.0 & $y_{112}$ \\
5 & 0.667 & 0.333 & 0.0 & $y_{122}$ \\
6 & 0.0 & 0.667 & 0.333 & $y_{223}$ \\
7 & 0.0 & 0.333 & 0.667 & $y_{233}$ \\
8 & 0.333 & 0.0 & 0.667 & $y_{133}$ \\
9 & 0.667 & 0.0 & 0.333 & $y_{113}$ \\
10 & 0.333 & 0.333 & 0.333 & $y_{123}$ \\
\hline
\end{tabular}

Simplex-lattice design was used to study the effect of the composition on the properties of ferrites, requiring a minimum number of experiments to study the influence of factors on the selected response functions [11]. The molar concentrations of cobalt, nickel, and zinc cations, respectively, were chosen as factors $\mathrm{x}_{1}, \mathrm{x}_{2}$, and $\mathrm{x}_{3}$. The design of the experiment is shown in Table 1 .

The upper and lower limits of each component were distributed as follows:

$$
\begin{array}{r}
0 \leq x_{1} \leq 0.33(\%), \\
0 \leq x_{2} \leq 0.33(\%), \\
0 \leq x_{3} \leq 0.33(\%), \\
x_{1}+x_{2}+x_{3}=0.33(\%) .
\end{array}
$$

Iron cation content is $0.67(\%)$. Three components of the model recipes changed simultaneously.

When studying the properties of a mixture, depending on the content of the components in it, the factor space can be represented as a regular simplex. An example of a simplex in two-dimensional space is a regular triangle.

For mixtures, the following relation holds: $\sum_{i=1}^{N} x_{i}=1$, where $x_{i} \geq 0$ is the content of components; $N$ is the number of components.

If at each vertex of the simplex we take the content of one of the components of the mixture as 1 , then in the abovementioned normalization condition, all the points located inside the two-dimensional regular simplex whose number of vertices equals the number of components of the mixture will satisfy. For example, in our case, this simplex is an equilateral triangle.

To each point of such a simplex, there corresponds a mixture of the corresponding composition, and any combination of the relative content of the components corresponds to a specific point on the simplex.

When planning the experiment in the form of "composition-property" diagrams, it is assumed that the property under investigation is a continuous function of the argument and is described with sufficient accuracy by the 

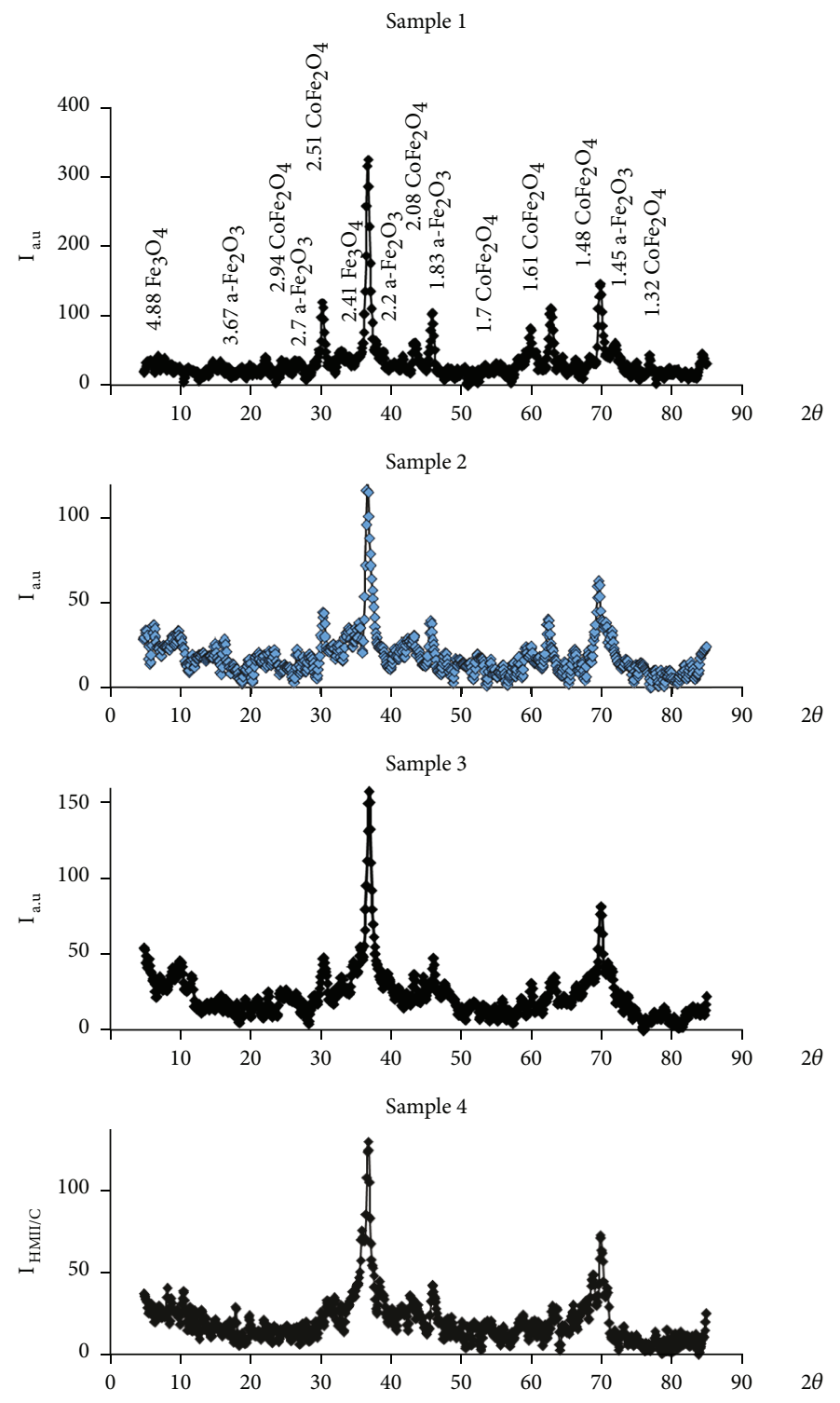

Figure 1: X-ray patterns of samples 1-4 (Table 1).

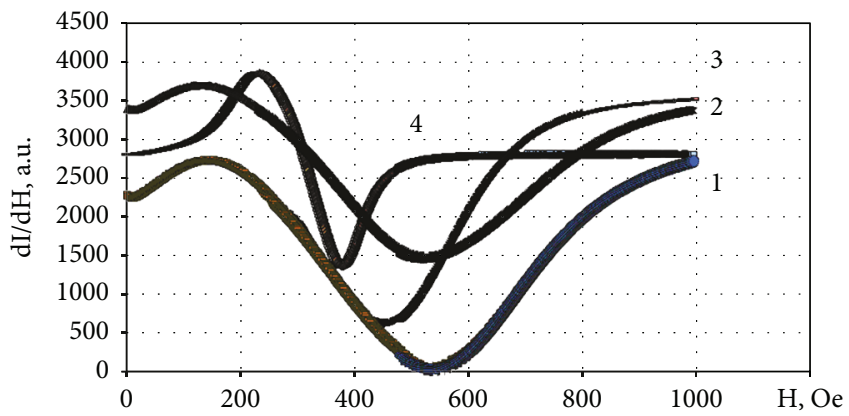

Figure 2: EPR spectra for samples 1-4 (Table 1).

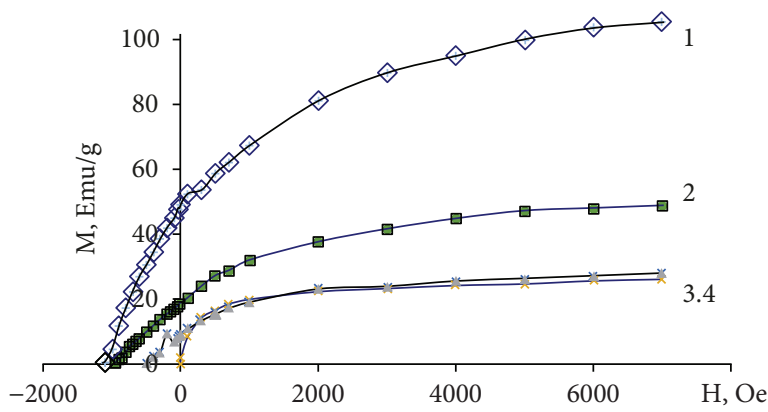

Figure 3: Magnetization curves of samples 1-4 (Table 1). 
TABle 2: Characteristics of Co-Zn-Ni ferrites.

\begin{tabular}{|c|c|c|c|c|c|c|c|}
\hline № & Composition & $\mathrm{H}_{\mathrm{c}}$ & $\mathrm{M}_{\mathrm{s}}$ & $\mathrm{H}_{\mathrm{R}}(\mathrm{mT})$ & I (a.u) & $\Delta \mathrm{Hpp}(\mathrm{mT})$ & $\mathrm{a}(\mathrm{A})$ \\
\hline 1. & $\mathrm{CoFe}_{2} \mathrm{O}_{4}$ & 1124 & 105.41 & 547 & 2700 & 398.7 & 8.35160 \\
\hline 2. & $\mathrm{Co}_{0.667} \mathrm{Ni}_{0.333} \mathrm{Fe}_{2} \mathrm{O}_{4}$ & 955 & 48.76 & 530 & 2242 & 383.65 & 8.34111 \\
\hline 3. & $\mathrm{Co}_{0.333} 7 \mathrm{Ni}_{0.667} \mathrm{Fe}_{2} \mathrm{O}_{4}$ & 503 & 27.80 & 445 & 3325 & 384 & 8.34016 \\
\hline 4. & $\mathrm{NiFe}_{2} \mathrm{O}_{4}$ & 2 & 26.05 & 364 & 2429 & 141.5 & 8.32012 \\
\hline 5. & $\mathrm{Ni}_{0.667} \mathrm{Zn}_{0.333} \mathrm{Fe}_{2} \mathrm{O}_{4}$ & 7 & 19.00 & 359 & 3824 & 63 & 8.37950 \\
\hline 6. & $\mathrm{Ni}_{0.333} \mathrm{Zn}_{0.667} \mathrm{Fe}_{2} \mathrm{O}_{4}$ & 9 & 7.70 & 345 & 3693 & 29.71 & 8.42310 \\
\hline 7. & $\mathrm{ZnFe}_{2} \mathrm{O}_{4}$ & 19 & 3.93 & 342 & 3008 & 21.83 & 8.36890 \\
\hline 8. & $\mathrm{Co}_{0.333} \mathrm{Zn}_{0.667} \mathrm{Fe}_{2} \mathrm{O}_{4}$ & 1 & 37.26 & 382 & 2538 & 156 & 8.37950 \\
\hline 9. & $\mathrm{Co}_{0.667} \mathrm{Zn}_{0.333} \mathrm{Fe}_{2} \mathrm{O}_{4}$ & 70 & 74.94 & 501 & 1121 & 366 & 8.34870 \\
\hline 10. & $\mathrm{Co}_{0.333} \mathrm{Zn}_{0.333} \mathrm{Ni}_{0.333} \mathrm{Fe}_{2} \mathrm{O}_{4}$ & 37 & 5.37 & 358 & 3189 & 122 & 8.38530 \\
\hline
\end{tabular}

$\mathrm{H}_{c}$ is the coercive field; $\mathrm{M}_{s}$ is the saturation magnetization; $\mathrm{H}_{\mathrm{R}}$ is the resonance field of the EPR spectrum, $m$ T; $\Delta \mathrm{H}_{\mathrm{p}}$ is the line width between the points of maximum slope on the EPR spectrum, $\mathrm{mT}$; I is the intensity of the EPR line of the spectrum, a.u; a is the lattice parameter, $\mathrm{A}$.

polynomial. The response surfaces in multicomponent systems have a complicated form and, for an adequate description of them, the necessary polynomials of a high degree.

For three-component mixtures, we write down a possible polynomial $(n=3)$

$$
\begin{aligned}
y= & \beta_{1} x_{1}+\beta_{2} x_{2}+\beta_{3} x_{3}+\beta_{12} x_{1} x_{2}+\beta_{13} x_{1} x_{3} \\
& +\beta_{23} x_{2} x_{3}+y_{12} x_{1} x_{2}\left(x_{1}-x_{2}\right)+y_{13} x_{1} x_{3}\left(x_{1}-x_{3}\right) \\
& +y_{23} x_{3} x_{2}\left(x_{2}-x_{3}\right)+\beta_{1_{23}} x_{1} x_{2} x_{3} .
\end{aligned}
$$

Calculation of the coefficients in the regression equation and checking its adequacy were carried out using the program STATISTICA 12.

The response surface in the composition-property diagrams was represented using isolines. The response functions were coercive field $\left(\mathrm{H}_{\mathrm{c}}\right)$, saturation magnetization $\left(\mathrm{M}_{\mathrm{s}}\right)$, resonant field $\left(\mathrm{H}_{\mathrm{R}}\right)$, width of the EPR peak $\left(\Delta \mathrm{H}_{\mathrm{pp}}\right)$, and intensity of the EPR peak of the spectrum (I, a.u.).

\section{Results and Discussion}

The magnetic properties of ferrites obtained under the action of CNP on the suspension of iron(II) and Me(II) polyhydroxides complexes are dependent on the $\mathrm{pH}$ of the solution of the iron(II) salt or the $\mathrm{Fe}(\mathrm{OH})_{2}$ suspension, the temperature of the reaction medium, the rate of oxidation, its activity and efficiency distribution in the reaction medium, and the concentration of iron(II) ions in the solution or iron(II) hydroxide in suspension [12-15]. But one of the most important factors is the cationic composition of ferrites [16-22]. In accordance with the simplex method, ten samples were synthesized and their properties were investigated.

Better samples are shown in Figures 1, 2, and 3. All results are shown in Table 2.

Mathematical processing of the experimental data using the program STATISTICA 12 allowed obtaining regression equations adequately describing the relationship between the magnetic indices and the composition of prototypes.

$$
\begin{aligned}
I_{\tilde{n}}= & 1124.0 x_{1}+749.81 x_{1} x_{2}-2409.19 x_{1} x_{3} \\
& +523.69 x_{1} x_{2}\left(x_{1}-x_{2}\right)-2023.50 x_{1} x_{3}\left(x_{1}-x_{3}\right) \\
& -4305.38 x_{1} x_{2} x_{3}, \\
M_{\mathrm{s}}= & 106 x_{1}+26 x_{2}-125 x_{1} x_{2}+57911 x_{2} x_{3}-174445 x_{1} x_{2} x_{3} \\
& -173732 x_{2} x_{3}\left(x_{2}-x_{3}\right) .
\end{aligned}
$$

The resulting regression equations were used to construct isolines of the magnetic characteristics of ferrites in the factorial space under study (Figures 3 and 4).

The highest value of the coercive field corresponds to the composition containing the maximum number of cobalt cations. An increase in the content of cobalt cations leads to an increase in the coercive field in all compositions. A positive effect of nickel cations on the saturation magnetization of ferrites along the side of the triangle $\mathrm{Ni}-\mathrm{Zn}$ and opposite on the Ni-Co side was also observed (Figure 4).

Moreover, the value of the saturation magnetization depends more on the content of cobalt cations. The highest magnetic indices correspond to the maximum content of cobalt. Thus, magnetic ferrites with an increased coercive field correspond to compositions 1,2,3, and magnetic ferrites with low coercive field 4,5,6,7. In the diagrams, an equilateral triangle with coordinates of the vertices of Co $(1,0,0)-\mathrm{Ni}$ $(0.75,0,0)-\mathrm{Zn}(0.25,0,0)$ can be identified, which corresponds to a region of higher values of the saturation magnetization.

Comparison of the main characteristics on the EPR spectra with magnetic properties makes it possible to explain the mechanism of action and to establish the contribution of the presence of ferrimagnetic cations and the degree of inversion of spinel. X-ray phase analysis showed that the samples contain the ferromagnetic phase probably $\mathrm{MeFe}_{2} \mathrm{O}_{4}$ and antiferromagnetic $\alpha-\mathrm{Fe}_{2} \mathrm{O}_{3}$. 


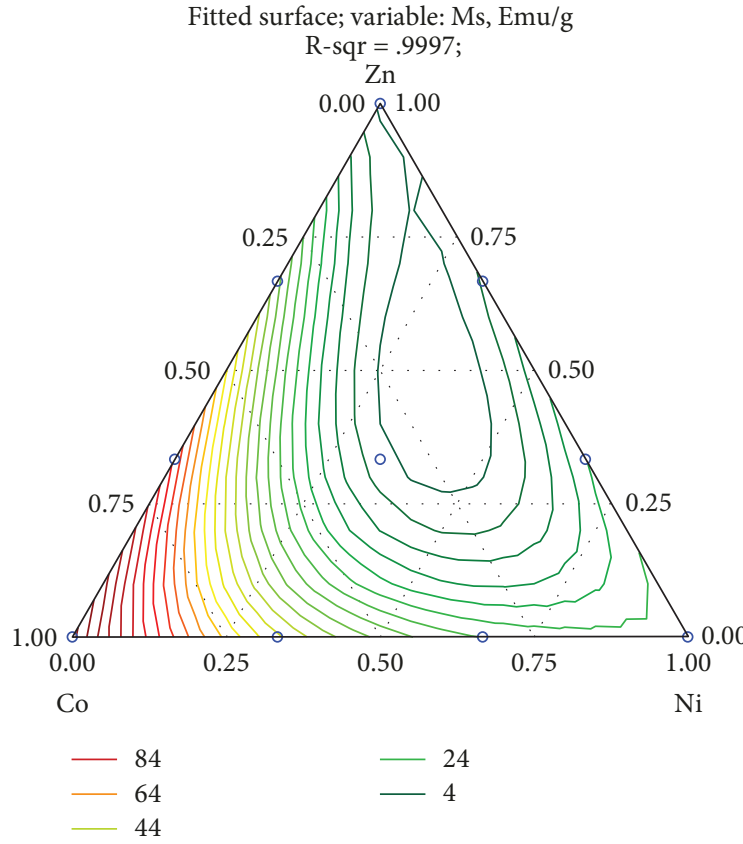

(a)

Fitted surface; variable: $\mathrm{Hc}$, Oe

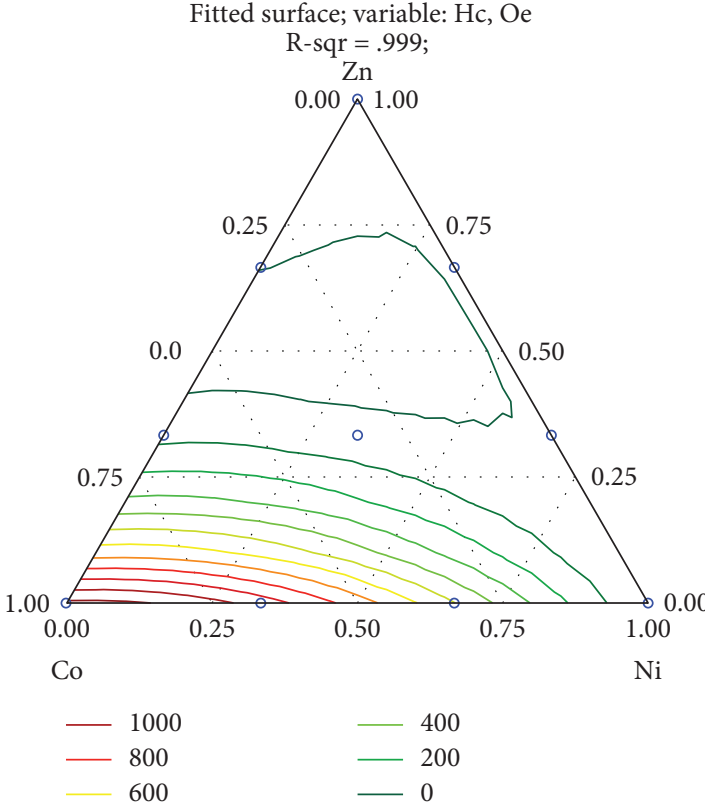

(c)

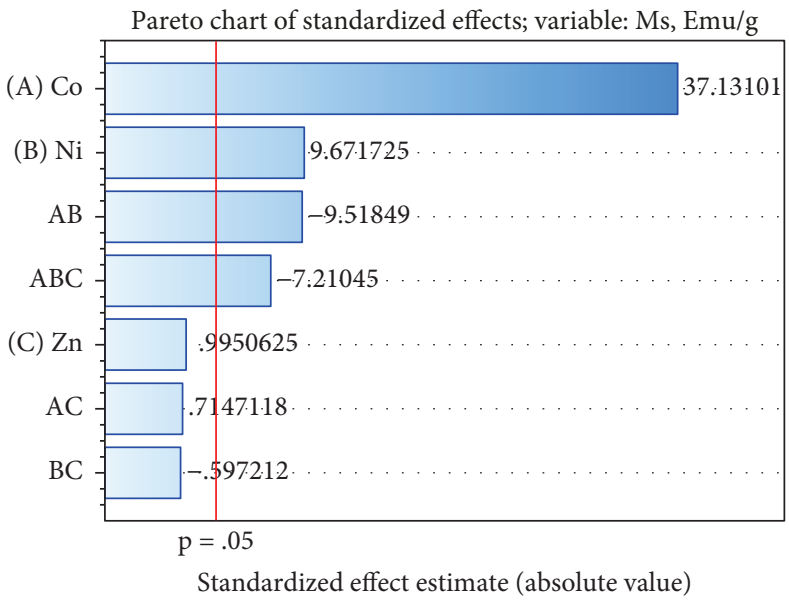

(b)

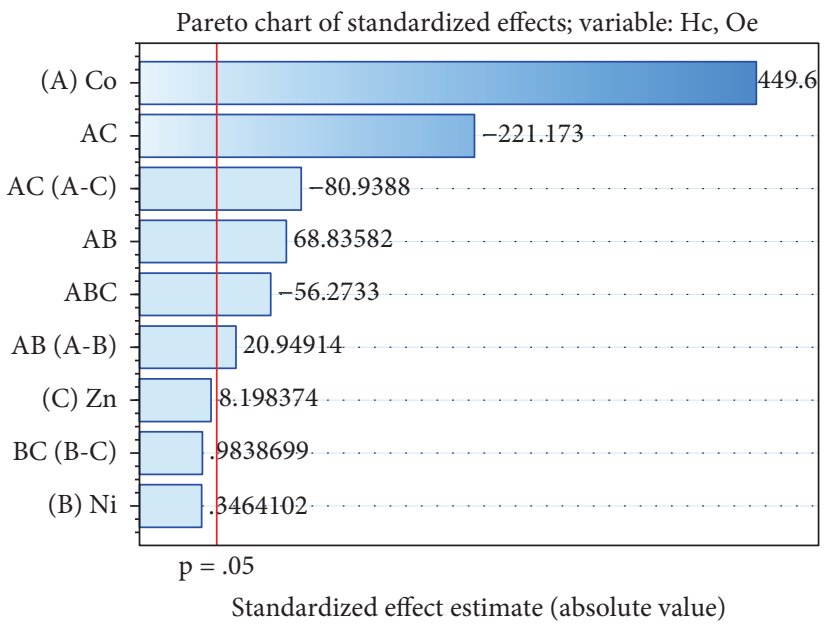

(d)

FIgure 4: Dependence of the saturation magnetization (a) and the coercive field (c) on the composition and the corresponding Pareto diagrams $((b),(d))$.

The magnetic characteristics correspond to the data of Xray phase analysis and EPR data (Figures 4 and 5).

All EPR spectra have a symmetric broad resonance signal, but their line width $\left(\Delta \mathrm{H}_{\mathrm{pp}}\right)$ and resonant magnetic field $\left(\mathrm{H}_{\mathrm{R}}\right)$ are very different (Table 2). It can be seen from Figures 5(a) and 5(b) that there is an increase in the resonance field and a change in the line width with an increase in the molar concentration of cobalt and nickel cations. It is seen that $\Delta \mathrm{H}_{\mathrm{pp}}$ is narrow; the intensity of the peaks is larger for a higher concentration of $\mathrm{Zn}$. The spectrum of the cobalt ferrite sample shows a rather wide signal $\left(\Delta \mathrm{H}_{\mathrm{pp}}=398.7 \mathrm{mT}\right)$. It is interesting that the effect of cobalt cations on the main characteristics of the EPR spectrum is much more significant than that of nickel. Consider the equation [21]

$$
\Delta H_{\mathrm{pp}}=\frac{K_{1}}{2 M_{\mathrm{s}}}+4 \pi M_{\mathrm{s}} \frac{p}{1-p}+H_{\mathrm{e}}+H_{\mathrm{id}}
$$




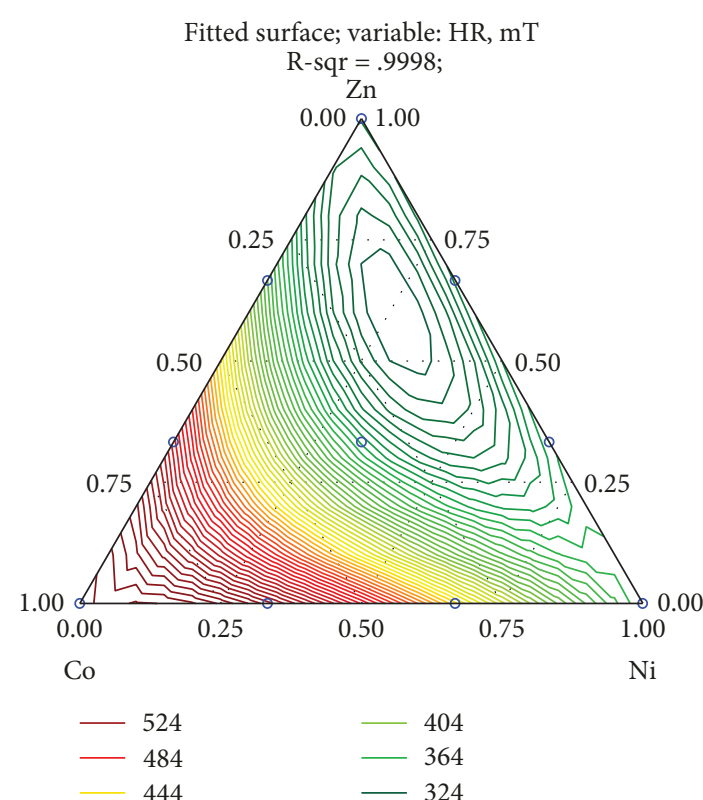

(a)

Fitted surface; variable: I, a.u. $\mathrm{R}$-sqr $=.9915$

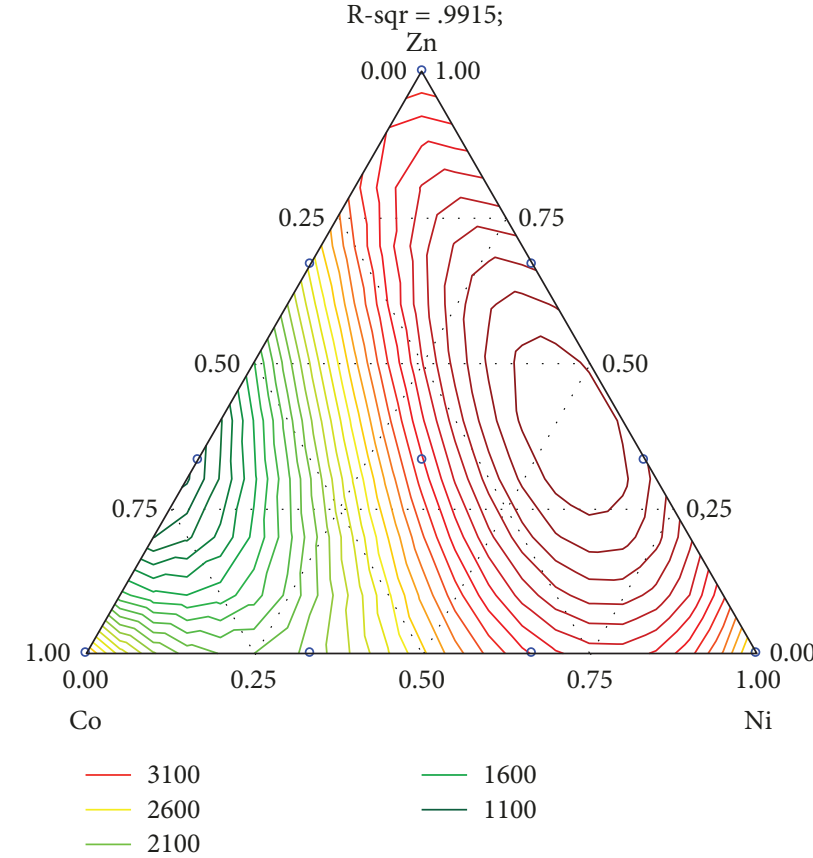

(c)

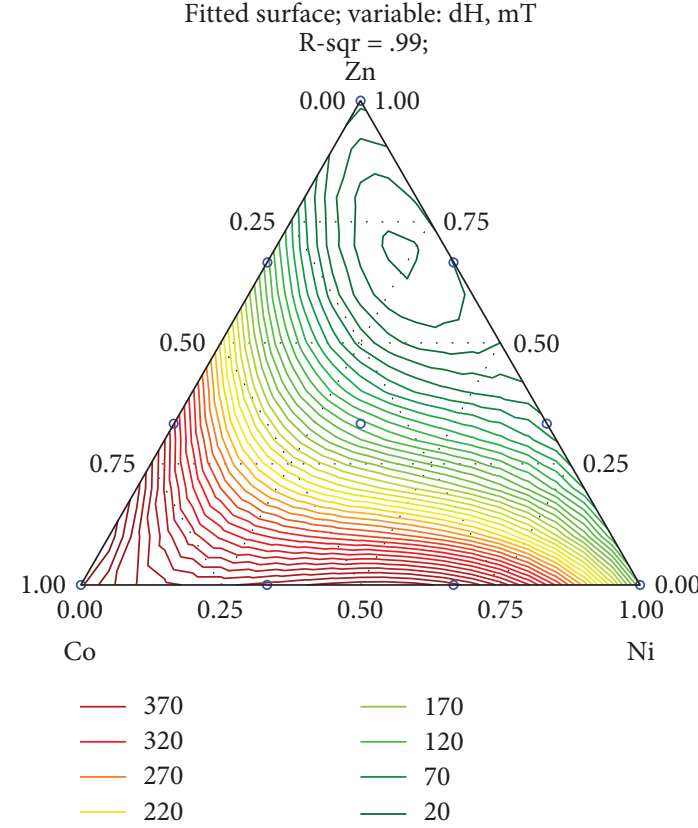

(b)

Fitted surface; variable: $\mathrm{a}, \mathrm{nm}$ $\mathrm{R}$-sqr $=.9981$;

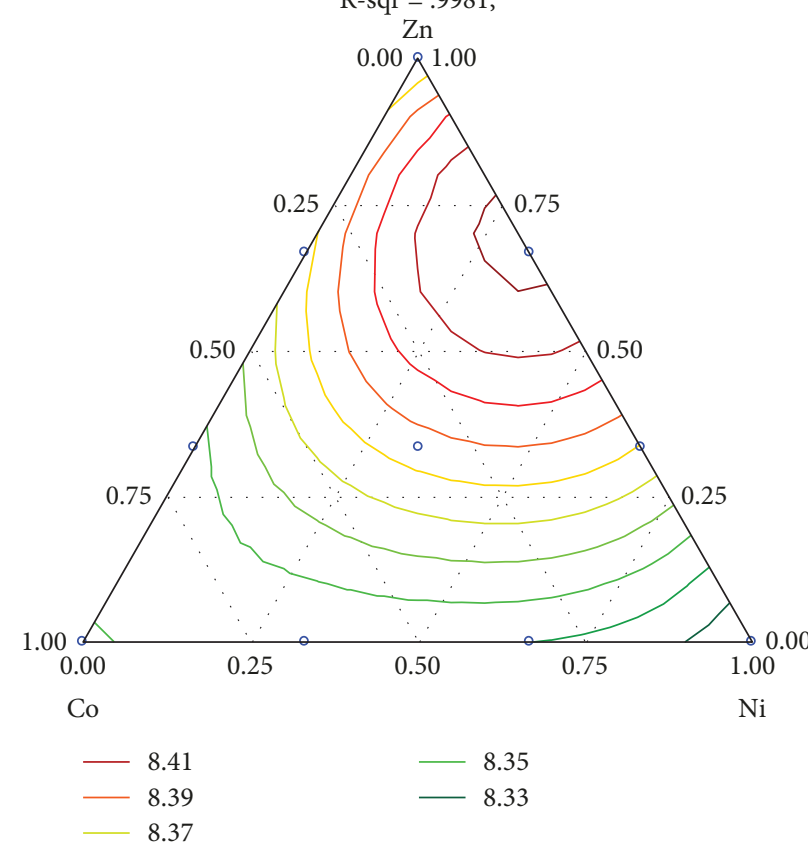

(d)

FIGURE 5: Dependence of the resonant field $\mathrm{H}_{\mathrm{R}}$ (a), the width of the ESR peak $\Delta \mathrm{H}_{\max }$ (b), the intensity of the ESR peak of the spectrum I (c), and the lattice parameter of the composition $(\mathrm{d})$.

where $\Delta H_{\mathrm{pp}}$ is the width of the EPR line of the spectrum, $K_{1}$ is the anisotropy, $p$ is the porosity, $H_{\mathrm{e}}$ is the noneddy currents, and $H_{\text {id }}$ is the inhomogeneous demagnetization.

Earlier studies have shown that the magnetic parameters of ferrites, in the system $\mathrm{CoO}-\mathrm{NiO},-\mathrm{ZnO}$, depend on the composition. An increase in the cobalt content in the system leads to an increase in the coercive field and the saturation magnetization. The increase in the content of cobalt cations in ferrites from 0 to $1.0 \mathrm{~mol}$. shares causes a significant increase in the coercive field from 23 to 1140 Oe. This fact is confirmed by a shift in the values of the lattice parameter $\mathrm{d}(8.35 \mathrm{~A})$ to a region of lower values $(8.32 \mathrm{~A})$ as well as an increase in the bandwidth on the EPR spectrum. 
From the values of coercivity $\left(\mathrm{H}_{\mathrm{c}}\right)$ and saturation magnetization $\left(M_{s}\right)$, the value of the anisotropy constant $K_{1}$ can be calculated using the following relation:

$$
K_{1}=\frac{H_{\mathrm{c}} M_{\mathrm{s}}}{0.96}
$$

Then

$$
\Delta H_{\mathrm{pp}}=\frac{H_{\mathrm{c}}}{1.82}+4 \pi M_{\mathrm{s}} \frac{p}{1-p}+H_{\mathrm{e}}+H_{\mathrm{id}}
$$

Taking into account that the largest value of the anisotropy constant corresponds to cobalt-containing ferrites, the contribution of the first term to equation (6) is the greatest. This determines a fairly broad peak of the EPR spectrum for samples 1-3.

The composition-property diagrams for the value of the resonance field and the line width correlate with the diagram for magnetic saturation. The resonant magnetic field increases with increasing content in cobalt and nickel samples. Reducing the width of the line, i.e., the narrowing of the derivative of the resonance signal with increasing content of $\mathrm{Zn}^{2+}$ and $\mathrm{Ni}^{2+}$ is associated with various causes. For zinc cations, first of all, these are their diamagnetic properties. For $\mathrm{Ni}^{2+}$ ions, this can be caused by their redistribution along sublattices and a decrease in the magnetic moment of the sublattice $\mathrm{B}$, taking into account the vacancies formed. This causes a general decrease in the magnetic moment. In accordance with this, a decrease in the resonant field for nickel ferrite occurs in accordance with formula

$$
H_{\mathrm{r}}=2 \omega \frac{\left(M_{\Sigma}\right)_{\mathrm{A}}-\left(M_{\Sigma}\right)_{\mathrm{B}}}{\left(M_{\mathrm{sp}}\right)_{\mathrm{A}}-\left(M_{\mathrm{sp}}\right)_{\mathrm{B}}}
$$

With an increase in the content of zinc cations, an increase in the intensity of the peaks and their narrowing are observed. Since the anisotropy constant for zinc ferrite is the smallest, it can be assumed that this is primarily due to the decrease of the first term in equation (6); the second term is also small, so the total value is also small. In these systems, the concentration of diamagnetic $\mathrm{Zn}^{2+}$ ions plays a decisive role. The existing dependence of $\mathrm{H}_{\mathrm{pp}}$ on the concentration of $\mathrm{Zn}$ is due to the superexchange interaction between $\mathrm{Ni}^{2+}$ and $\mathrm{Fe}^{2+}$ through nonmagnetic $\mathrm{O}^{2-}$ ions.

Almost complete coincidence of the isolines for the graphs $\mathrm{M}_{\mathrm{s}}=\mathrm{f}(\mathrm{Ni}, \mathrm{Co}, \mathrm{Zn})$ and $\mathrm{H}_{\mathrm{r}}=\mathrm{f}(\mathrm{Ni}, \mathrm{Co}, \mathrm{Zn})$ makes it possible to assume that the main factor determining the ferrite magnet is the cation distribution over the sublattices with allowance for the concentration of diamagnetic ions.

\section{Conclusions}

The article is devoted to the analysis of changes in the magnetic characteristics of ferrites in the $\mathrm{Fe}_{2} \mathrm{O}_{3}-\mathrm{CoO}-\mathrm{ZnO}$ system by the simplex method. Ferrites of Ni-Zn, Co-Zn, and $\mathrm{Co}-\mathrm{Ni}$ were synthesized in the form of nanoparticles using a new method for processing contact nonequilibrium low-temperature plasma. The crystalline, magnetic, and microstructure of the finished crystallites were elucidated using several methods. The macroscopic characteristics of magnetic materials are inherently rooted in their atomic structure. Understanding the crystal structure is necessary for the synthesis of magnetic nanomaterials with optimal properties. For spinel ferrites, in particular, the choice of a bivalent cation and its distribution between the tetrahedral and octahedral sites directly determine their magnetic behavior. The effect of the mutual influence of the content of different cations on the saturation magnetization and the coercive field was investigated using the simplex-lattice method. A magnetic investigation using a vibrational magnetometer shows that under these synthesis conditions, low magnetization values for $\mathrm{Ni}-\mathrm{Zn}$ ferrites and high magnetization values for the whole Co- $\mathrm{Zn}$ and $\mathrm{Co}-\mathrm{Ni}$ ferrite series are observed. The EPR spectra show that the value of the resonant field and line width corresponds to the value of the magnetic saturation. In this work, a new method of synthesis of combustion is the nanoferrite used to produce $\mathrm{Ni}-\mathrm{Zn}$. The EPR spectra of ferrites are explained on the basis of superexchange interaction.

\section{Data Availability}

Previously described method simplex-lattice design was used to support this study and is available at https://books.google .com.ua/. This prior study is cited at the relevant place within the text as reference [11].

\section{Conflicts of Interest}

The authors declare that they have no competing interests.

\section{References}

[1] A. B. Gadkari, T. J. Shinde, and P. N. Vasambekar, "Ferrite gas sensors," IEEE Sensors Journal, vol. 11, no. 4, pp. 849-861, 2011.

[2] W. Hu, N. Qin, G. Wu, Y. Lin, S. Li, and D. Bao, “Opportunity of spinel ferrite materials in nonvolatile memory device applications based on their resistive switching performances," Journal of the American Chemical Society, vol. 134, no. 36, pp. 14658-14661, 2012.

[3] L. Živanov, M. Damnjanović, N. Blaž, A. Marić, M. Kisić, and G. Radosavljević, "Soft ferrite applications," in Magnetic, Ferroelectric, and Multiferroic Metal Oxides, pp. 387-409, Elsevier, 2018.

[4] V. M. Chernyshev and N. P. Shabelskaya, "Comparative analysis of catalytic activity in complex NiO-CuO-Fe $\mathrm{O}_{3}-\mathrm{Cr}_{2} \mathrm{O}_{3}$ Oxide system of different production technologies," Materials Science Forum, vol. 870, pp. 118-122, 2016.

[5] R. S. Melo, P. Banerjee, and A. Franco, "Hydrothermal synthesis of nickel doped cobalt ferrite nanoparticles: optical and magnetic properties," Journal of Materials Science: Materials in Electronics, vol. 29, no. 17, pp. 14657-14667, 2018.

[6] L. A. Frolova and A. A. Pivovarov, "Investigation of conditions for ultrasound-assisted preparation of nickel ferrite," High Energy Chemistry, vol. 49, no. 1, pp. 10-15, 2015.

[7] L. A. Frolov, A. A. Pivovarov, A. S. Baskevich, and A. I. Kushnerev, "Structure and properties of nickel ferrites 
produced by glow discharge in the $\mathrm{Fe}^{2+}-\mathrm{Ni}^{2+}-\mathrm{SO}_{4}{ }^{2-}-\mathrm{OH}^{-}$ system," Russian Journal of Applied Chemistry, vol. 87, no. 8, pp. 1054-1059, 2014.

[8] R. N. Bhowmik and K. S. Aneeshkumar, "Low temperature ferromagnetic properties, magnetic field induced spin order and random spin freezing effect in $\mathrm{Ni}_{1.5} \mathrm{Fe}_{1.5} \mathrm{O}_{4}$ ferrite; prepared at different $\mathrm{pH}$ values and annealing temperatures," Journal of Magnetism and Magnetic Materials, vol. 460, pp. 177-187, 2018.

[9] G. Avramovic-Cingara, J. Zweck, J. D. Giallonardo, G. Palumbo, and U. Erb, "Magnetic domain structure in nanocrystalline nickel electrodeposits," Journal of Applied Physics, vol. 123, no. 23, pp. 234301-234301, 2018.

[10] R. N. Bhowmik, R. Ranganathan, R. Nagarajan, B. Ghosh, and S. Kumar, "Role of strain-induced anisotropy on magnetic enhancement in mechanically alloyed $\mathrm{Co}_{0.2} \mathrm{Zn}_{0.8} \mathrm{Fe}_{2} \mathrm{O}_{4}$ nanoparticle," Physical Review B, vol. 72, no. 9, 2005.

[11] J. A. Cornell, Experiments with Mixtures: Designs, Models, and the Analysis of Mixture Data, vol. 403, John Wiley \& Sons, 2011.

[12] C. Singh, S. Bansal, and S. Singhal, "Synthesis of $\mathrm{Zn}_{1-\mathrm{x}} \mathrm{Co}_{\mathrm{x}-}$ $\mathrm{Fe}_{2} \mathrm{O}_{4}$ /MWCNTs nanocomposites using reverse micelle method: investigation of their structural, magnetic, electrical, optical and photocatalytic properties," Physica B: Condensed Matter, vol. 444, pp. 70-76, 2014.

[13] M. Sundararajan, L. John Kennedy, and J. Judith Vijaya, "Synthesis and characterization of cobalt substituted zinc ferrite nanoparticles by microwave combustion method," Journal of Nanoscience and Nanotechnology, vol. 15, no. 9, pp. 67196728, 2015.

[14] A. Manikandan, L. J. Kennedy, M. Bououdina, and J. J. Vijaya, "Synthesis, optical and magnetic properties of pure and Codoped $\mathrm{ZnFe}_{2} \mathrm{O}_{4}$ nanoparticles by microwave combustion method," Journal of Magnetism and Magnetic Materials, vol. 349, pp. 249-258, 2014.

[15] I. Zālīte, G. Heidemane, A. Krūmina, D. Rašmane, and M. Maiorov, " $\mathrm{ZnFe}_{2} \mathrm{O}_{4}$ containing nanoparticles: synthesis and magnetic properties," Materials Science and Applied Chemistry, vol. 34, no. 1, pp. 38-44, 2017.

[16] K. Sun, Z. Pu, Y. Yang et al., "Rietveld refinement, microstructure and ferromagnetic resonance linewidth of iron-deficiency NiCuZn ferrites," Journal of Alloys and Compounds, vol. 681, pp. 139-145, 2016.

[17] M. Su, C. Liao, T. Chan et al., "Incorporation of cadmium and nickel into ferrite spinel solid solution: X-ray diffraction and $\mathrm{X}$-ray absorption fine structure analyses," Environmental Science \& Technology, vol. 52, no. 2, pp. 775-782, 2018.

[18] T. R. Tatarchuk, N. D. Paliychuk, M. Bououdina et al., "Effect of cobalt substitution on structural, elastic, magnetic and optical properties of zinc ferrite nanoparticles," Journal of Alloys and Compounds, vol. 731, pp. 1256-1266, 2018.

[19] B. Thangjam and I. Soibam, "Structural, electrical and magnetic properties of $\mathrm{Mg}$ doped $\mathrm{Ni}-\mathrm{Cu}-\mathrm{Zn}$ nanoferrites synthesized by citrate precursor method," International Journal of Applied Engineering Research, vol. 12, no. 23, pp. 1320113206, 2017.

[20] M. T. Jamil, J. Ahmad, S. H. Bukhari et al., "Effect on structural and optical properties of Zn-substituted cobalt ferrite $\mathrm{CoFe}_{2} \mathrm{O}_{4}$," Journal of Ovonic Research, vol. 3, no. 1, pp. 4553,2017
[21] C. M. Srivastava and M. J. Patni, "Ferromagnetic relaxation processes in polycrystalline magnetic insulators," Journal of Magnetic Resonance, (1969), vol. 15, no. 2, pp. 359-366, 1974.

[22] R. N. Bhowmik, S. Kazhugasalamoorthy, R. Ranganathan, and A. K. Sinha, "Tuning of composite cubic spinel structure in $\mathrm{Co}_{1.75} \mathrm{Fe}_{1.25} \mathrm{O}_{4}$ spinel oxide by thermal treatment and its effects on modifying the ferromagnetic properties," Journal of Alloys and Compounds, vol. 680, pp. 315-327, 2016. 


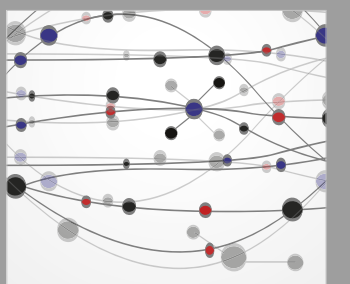

The Scientific World Journal
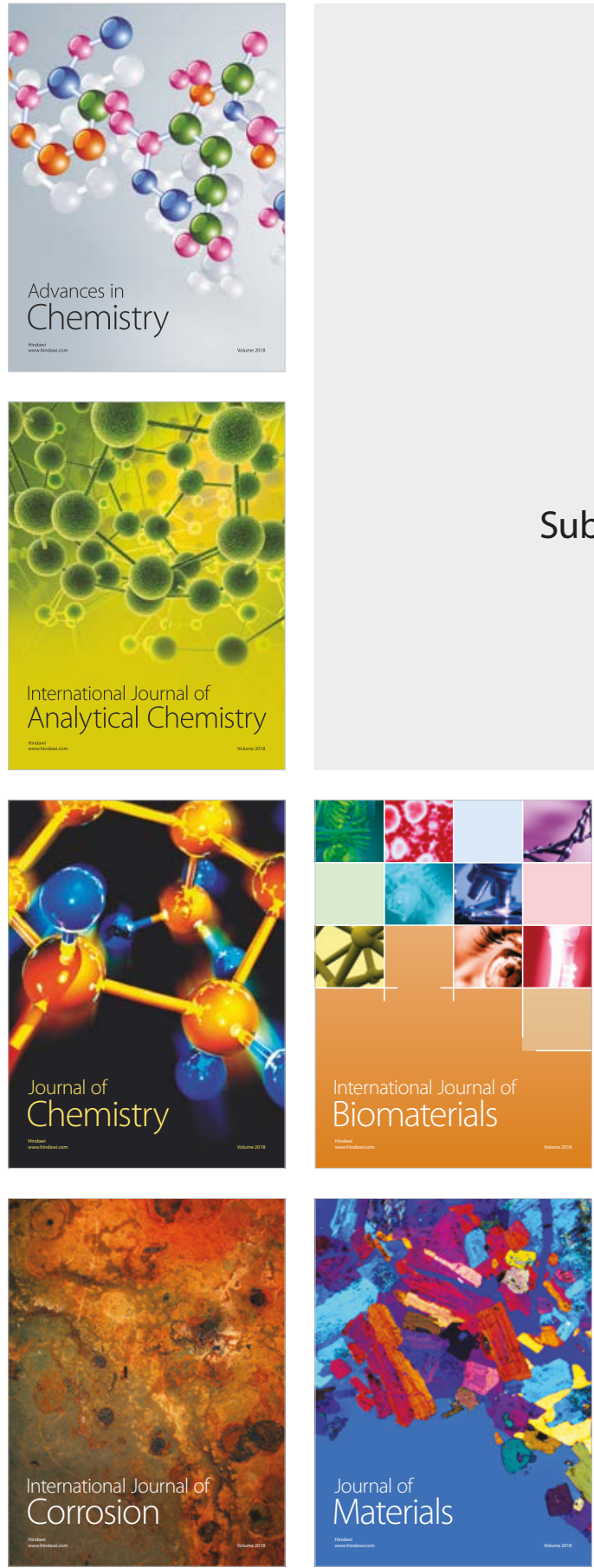

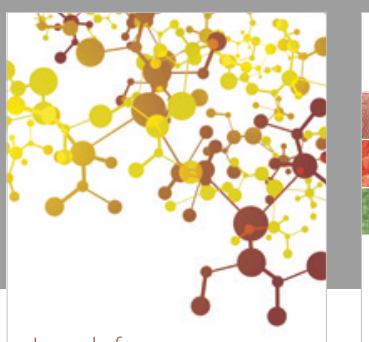

Journal of

Applied Chemistry
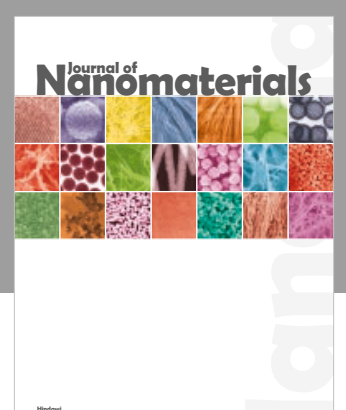

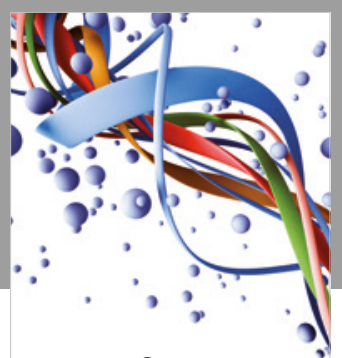

Scientifica

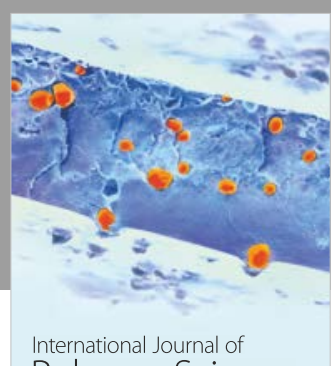

Polymer Science

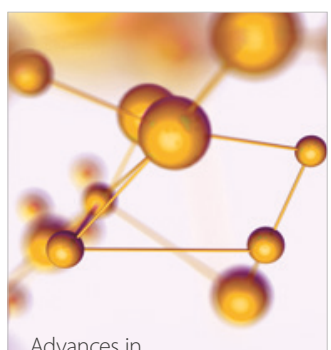

Physical Chemistry
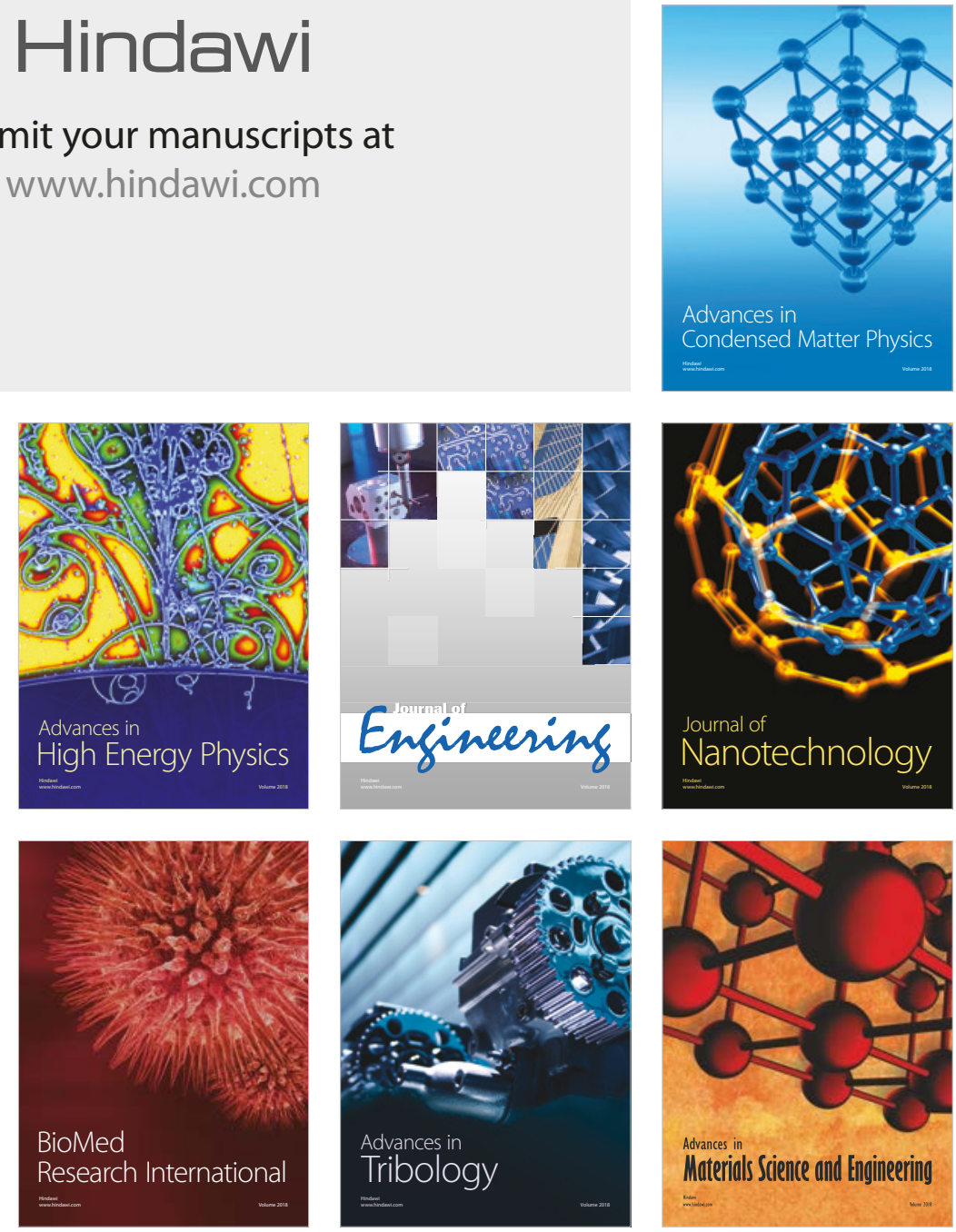